Ann. Zootech., 1984, 33 (2), 171-186

\title{
Détermination des besoins azotés de la chèvre en lactation
}

\author{
J. BRUN-BELLUT, G. BLANCHART et B. VIGNON \\ Laboratoire de Zootechnie, E.N.S.A.I.A., \\ 38, rue Sainte-Catherine, F 54000 Nancy
}

\section{Résumé}

A partir de 216 bilans azotés mesurés sur 67 chèvres âgées de 2 à 6 ans, entre la $1^{\text {re }}$ et la $40^{\mathrm{e}}$ semaine de lactation, nous avons déterminé le besoin azoté d'entretien et le rendement de la transformation de l'azote digéré en azote protéique du lait.

Nous avons utilisé 2 méthodes :

- méthode factorielle : le besoin azoté d'entretien est considéré comme égal à la somme de l'azote urinaire endogène (EUN) et de l'azote fécal métabolique (MFN) :

$\mathrm{EUN}=0,0434 \mathrm{PV}$

MFN $=0,0169$ CBND

Dans nos conditions, les pertes minimales d'azote sont au total de :

$$
\mathrm{EUN}+\mathrm{MFN}=0,25 \mathrm{~g} \mathrm{~N} / \mathrm{kg} \mathrm{P}^{0,75}
$$

- méthode globale : par régression, nous expliquons l'utilisation de l'azote digéré en fonction du poids métabolique, de la production d'azote protéique dans le lait, du bilan azoté, des pertes d'azote au niveau du rumen (estimées par la différence PDIN-PDIE) et de l'excès de l'azote digéré par rapport à son utilisation pour l'entretien et la production de lait (estimé par le taux d'urée du lait).

Cette méthode permet d'évaluer le besoin azoté d'entretien à $0,32 \mathrm{~g} / \mathrm{kg} \mathrm{P}^{0,75}$ en azote digestible. Le rendement de la transformation de l'azote digéré en azote protéique du lait varie entre 0,72 et 0,81 suivant les niveaux de couverture des besoins azoté et énergétique. Les plus forts rendements sont mesurés sur des animaux en bilan azoté positif et en bilan énergétique négatif.

Les fortes valeurs de rendement obtenues peuvent être expliquées par une économie d'azote par l'animal liée à la faiblesse de l'excrétion urinaire d'azote $(8,9 \mathrm{~g}$ d'azote par jour).

Chez les animaux en bilan azoté négatif, le rendement de la transformation de l'azote digéré en azote protéique du lait est de 0,56 ; il est inférieur au rendement de transformation de l'azote corporel mobilisé en azote protéique du lait $(0,71)$.

Mots clés : azote, chèvre, entretien, lait.

\section{Introduction}

Les besoins azotés ont été peu étudiés chez la chèvre en lactation (tabl. 1). Les données sont obtenues essentiellement sur mâles castrés par la méthode des bilans 


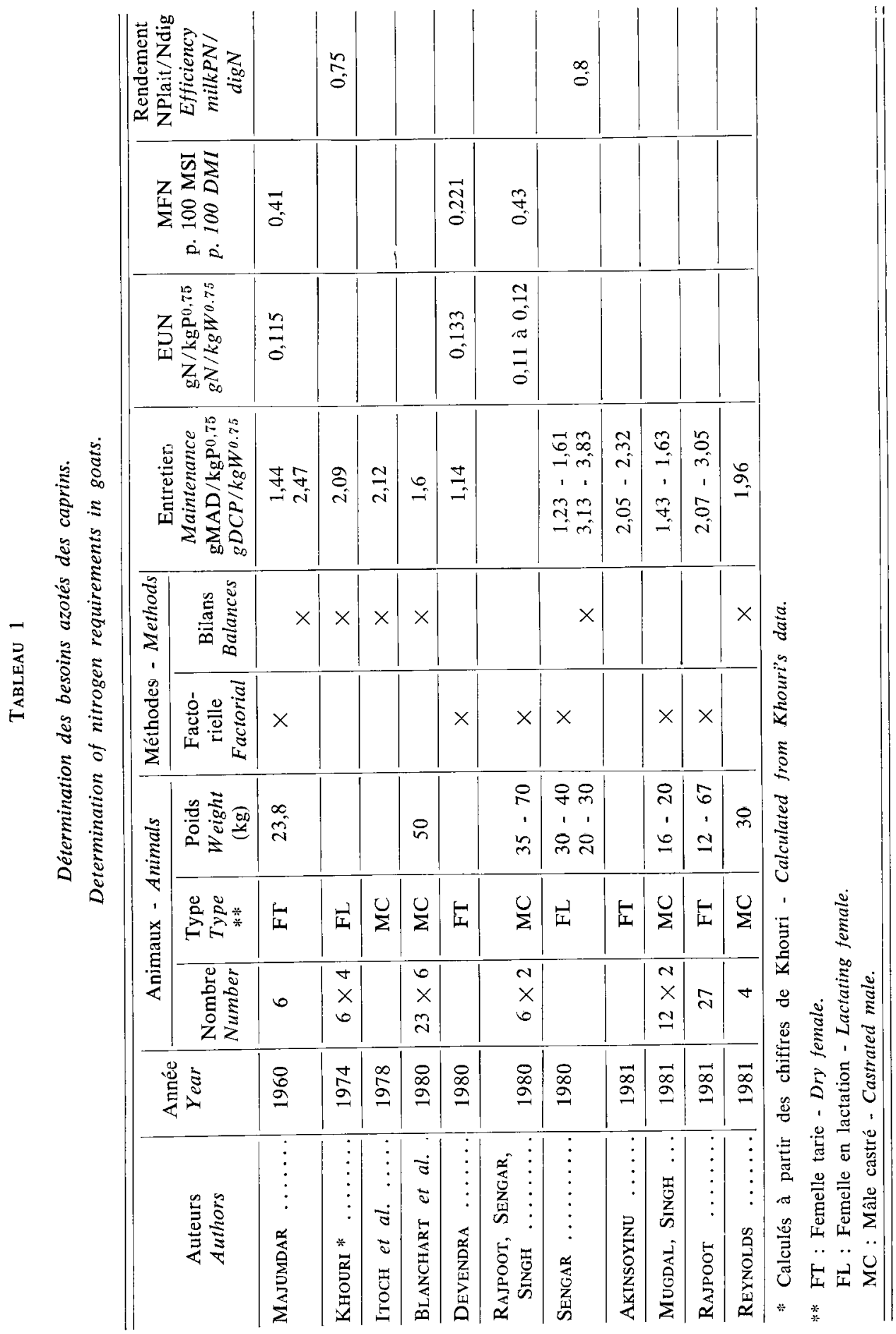


(Itoch et al., 1978, Blanchart, Brun-Bellut \& Vignon, 1980, et Reynolds, 1981), ou par la méthode factorielle en déterminant le plus souvent par régression l'azote urinaire endogène (EUN) et l'azote fécal métabolique (MFN) (Rajpoot, SENGar \& Singh, 1980, Mudgal \& Singh, 1981, Blanchart \& Brun-Bellut, 1982).

Des estimations des besoins d'entretien ont été effectuées sur chèvres par la méthode des bilans (Majumdar, 1960, Delort-Laval cité par Morand-Fenr \& Sauvant, 1978, Sengar, 1980, AKinsoyinu, 1981) et par la méthode factorielle (MAjumdar, 1960, Devendra, 1980, Sengar, 1980, et Rajpoot, 1981).

L'ensemble des résultats obtenus est compris entre 1,14 et $3,83 \mathrm{~g}$ de $\mathrm{MAD} / \mathrm{kg}$ $\mathrm{P}^{0,75}$. Les variations importantes entre les résultats ne dépendent ni du sexe ni du gabarit des animaux (tabl. 1). Les variations entre auteurs ne semblent pas imputables à la méthode employée (méthode des bilans ou méthode factorielle). Cependant, $\mathrm{MA}_{\mathrm{A}}$ JUMdar (1960) et SENGar (1980) qui ont utilisé simultanément les deux méthodes, ont trouvé, par la méthode des bilans, des besoins plus élevés que par la méthode factorielle.

Les valeurs maximales sont obtenues par Rajpoot, SEngar \& Singh (1980) sur boucs castrés $\left(2,07\right.$ à $3,05 \mathrm{~g}$ de MAD/ $\left.\mathrm{kg} \mathbf{P}^{0,75}\right)$ et par Sengar $(1980)$ sur chèvres $(3,13$ à $3,83 \mathrm{~g}$ de $\left.\mathrm{MAD} / \mathrm{kg} \mathrm{P}^{0,75}\right)$.

Les estimations simultanées des besoins d'entretien et du rendement de la transformation de l'azote apparemment digéré en azote protéique du lait chez la chèvre sont rares. La plupart des recommandations pour la production de lait des chèvres, comme celles de Morand-Fehr \& Sauvant (1978) utilisent les rendements obtenus chez la vache laitière qui sont compris entre 0,62 et 0,75 (Arc, 1965, KaUfmanN, 1976, Vérité \& Journet, 1978). Tamminga \& Oldham (1980) dans une revue bibliographique citent des rendements de transformation de l'azote apparemment digéré en azote protéique du lait compris entre 0,60 et $0,83 \mathrm{chez}$ la vache laitière. SENGAR (1980) signale un rendement de 0,80 pour des chèvres laitières.

A partir de bilans azotés mesurés chez des chèvres en lactation nous avons calculé les besoins azotés de la chèvre laitière en production par la méthode factorielle et par la méthode globale des bilans.

\section{Matériel et méthodes}

\section{A. Animalıx}

216 bilans azotés ont été mesurés sur 67 chèvres multipares de race Alpine Chamoisée en 5 séries de mesures :

- 15 chèvres pendant les 7 premières semaines de lactation (en continu);

- 12 chèvres pendant les 4 premières semaines de lactation (en continu);

- 31 chèvres pendant 2 ou 3 semaines, à partir de la $12^{\circ}$ ou de la $16^{\circ}$ semaine de lactation ( 2 séries en continu);

- 9 chèvres en $40^{\prime \prime}$ semaine de lactation.

Les numéros de lactation des chèvres sont compris entre 2 et 6 . 


\section{B. Alimentation}

Les rations distribuées sont présentées au tableau 2. Elles comprennent des aliments déshydratés (pulpes de betteraves ou Ray Grass) et des aliments concentrés (orge, tourteaux de soja ou de colza formolés ou non, avec ou sans urée). Les animaux disposent en outre de paille de blé distribuée à volonté. La consommation de paille, mesurée chaque jour, ne représente jamais plus de 5 p. 100 de la matière sèche totale ingérée.

Tableau 2

Régimes (p. 100 de MS totale)

Diets (p. 100 of whole DM)

\begin{tabular}{|c|c|c|c|c|}
\hline \multirow{2}{*}{\multicolumn{2}{|c|}{$\begin{array}{l}\text { Pulpes de betteraves déshydratées } \ldots \ldots \ldots \ldots \ldots \ldots \ldots \ldots \\
\text { Dehydrated sugar beet pulps } \\
\text { Ray grass déshydraté } \ldots \ldots \ldots \ldots \ldots \ldots \ldots \ldots \ldots \ldots \ldots \ldots \\
\text { Dehydrated ray grass }\end{array}$}} & \multicolumn{2}{|c|}{$70-75$} & - \\
\hline & & \multicolumn{2}{|c|}{ 一 } & $60-70$ \\
\hline \multicolumn{2}{|l|}{$\begin{array}{l}\text { Orge } \\
\text { Barley }\end{array}$} & \multicolumn{2}{|c|}{$10-20$} & $15-25$ \\
\hline \multirow{4}{*}{$\begin{array}{l}\text { Tourteaux } \\
\text { Meals }\end{array}$} & $\begin{array}{l}\text { Soja }+ \text { colza } \ldots \ldots \ldots \ldots \\
\text { Soybean }+ \text { rapeseed }\end{array}$ & $5-17$ & - & - \\
\hline & $\begin{array}{l}\text { Soja }+ \text { colza } \ldots \ldots \ldots \ldots \ldots \text { F } \\
\text { Soybean }+ \text { rapeseed }\end{array}$ & $0-12$ & - & - \\
\hline & $\begin{array}{l}\text { Soja }+ \text { colza }+ \text { urée } \ldots \ldots \text { F F } \\
\text { Soybean }+ \text { rapeseed }+ \text { urea }\end{array}$ & - & $4-20$ & 一 \\
\hline & $\begin{array}{l}\text { Soja }+ \text { urée } \ldots \ldots \ldots \ldots \ldots \ldots \\
\text { Soybean }+ \text { urea }\end{array}$ & - & - & 15 \\
\hline
\end{tabular}

F : Traité au formol - Formaldehyde treated.

En première semaine de lactation, les besoins sont calculés à partir de la production maximale de la lactation antérieure, avec une correction permettant de tenir compte du numéro de lactation. Dès la deuxième semaine de lactation, les apports d'énergie et d'azote sont ajustés en fonction des exportations mesurées la semaine précédente. Les normes utilisées sont celles présentées par MORAND-FEHR \& SAUVANT (1978).

\section{Contrôles, prélèvements et analyses}

Pour chaque chèvre, sont mesurées la consommation et les productions journalières de fèces et d'urine; des échantillons représentatifs des aliments, des refus, des fèces et des urines sont prélevés tous les jours. Les échantillons sont regroupés 
par période de mesure avant d'être analysés : un regroupement par aliment et un regroupement (en proportion des quantités produites) pour les refus et pour les fèces de chaque individu. Les échantillons individuels d'urine sont analysés quotidiennement.

La production laitière est mesurée individuellement à chaque traite et un échantillon du lait produit par chèvre et par traite est prélevé pour être analysé quotidiennement.

\section{Méthodes de calcul}

Les bilans azotés sont calculés pour chaque chèvre sur des périodes de 7 jours consécutifs, sauf pour la première période après la mise-bas dont la durée varie entre 4 et 10 jours, en fonction du jour de la semaine où a lieu la mise-bas. Le bilan azoté est calculé en prenant en compte l'azote ingéré et les exportations d'azote par les fèces, les urines et le lait :

$$
\mathrm{N}_{\text {bilan }}=\mathrm{N}_{\text {ingéré }}-\left(\mathrm{N}_{\text {fécal }}+\mathrm{N}_{\text {urinairè }}+\mathrm{N}_{\text {lait }}\right)
$$

Nous avons déterminé les besoins azotés par la méthode factorielle (besoins d'entretien) et par la méthode globale (besoins d'entretien et besoins de production).

\section{Méthode factorielle}

Détermination de l'azote urinaire endogène (EUN) et de l'azote fécal métabolique (MFN).

\section{$E U N$}

L'azote excrété dans l'urine provient :

- des pertes d'ammoniac au niveau du rumen, plus ou moins compensées par le recyclage d'urée dans l'ensemble du tube digestif;

- de l'azote digéré en excès par rapport à celui utilisé pour la production de lait et non retenu dans le pool azoté de l'animal; lait ;

- des pertes occasionnées par la transformation de l'azote digéré en azote du

- du remaniement du pool azoté de l'animal.

Lorsque les chèvres sont en bilan azoté négatif ou nul et lorsque leur ration ne contient pas de quantités excédentaires d'azote fermentescible, les pertes d'azote par l'urine sont essentiellement dues à la transformation de l'azote digéré en azote du lait et à l'azote urinaire endogène.

Nous établissons l'équation de régression suivante :

où $\mathrm{PV}$ est le poids vif des animaux.

$$
\mathrm{N}_{\text {urinaire }}=\mathrm{a} \mathrm{N}_{\text {digéré }}+\mathrm{bPV}+\mathrm{c}
$$

Dans cette équation, b PV $+c$ représente l'azote urinaire endogène. 


\section{$M F N$}

Différents modèles permettant d'expliquer les variations de la production d'azote fécal ont été présentés récemment (Blanchart, BRUn-Bellut \& VignoN, 1983, Giger \& SaUVANT, 1983).

Le modèle que nous avons proposé permet une estimation des pertes fécales d'azote de différentes origines :

- l'azote alimentaire non dégradé est estimé à partir de la quantité de matières azotées insolubles ingérée $\left(\mathrm{MA}_{\text {ins. }}\right)$;

- l'azote microbien d'origine alimentaire est estimé à partir de la quantité de matière organique digestible ingérée (MOD);

- l'azote endogène est estimé à partir de la cellulose non digestible ingérée (CBND). La variable CBND a été choisie parce qu'elle est représentative de l'importance du transit digestif et, par conséquent, des pertes d'azote endogène qu'il occasionne (desquamations, sécrétions). De plus, contrairement à la matière sèche ingérée, variable utilisée par de nombreux auteurs, elle est indépendante des autres variables que nous avons retenues.

Le modèle retenu est le suivant :

$$
\mathrm{N}_{\text {Fécal }}=\mathrm{a} \mathrm{MA}_{\mathrm{ins}}+\mathrm{b} \mathrm{MOD}+\mathrm{c} \text { CBND }
$$

Dans cette équation l'azote fécal métabolique est donc $\mathrm{MFN}=\mathrm{c}$ CBND.

Les pertes obligatoires d'azote, correspondant au besoin d'entretien sont égales à MFN + EUN.

\section{Méthode globale}

L'azote digéré est utilisé pour l'entretien (proportionnellement au poids métabolique) pour la production laitière (proportionnellement à la production d'azote protéique dans le lait) et pour l'augmentation éventuelle du pool azoté (proportionnellement au bilan azoté). Des pertes peuvent survenir :

- au niveau du rumen par absorption d'ammoniac et élimination urinaire de l'azote correspondant (proportionnelles à la différence PDIN $-\mathrm{PDIE}=\Delta \mathrm{PDI}$ ) ;

- dans l'urine quand l'animal ingère de l'azote digestible en excès par rapport à ses besoins. Nous avons montré par ailleurs (Brun-Bellut, LAurent \& Vignon, 1983) que le taux d'urée du lait (TUL) est un bon estimateur de l'excès d'azote digestible ingéré par rapport aux exportations dans le lait.

Nous avons donc établi l'équation de régression suivante pour expliquer le devenir de l'azote digéré :

$$
\mathrm{N}_{\text {digéré }}=\mathrm{a} \mathrm{NP}_{\text {lait }}+\mathrm{b} \mathrm{P}^{0,75}+\mathrm{c} \mathrm{N}_{\text {bilan }}+\mathrm{d} \Delta \text { PDI }+\mathrm{e} \text { TUL }
$$

Cette équation de régression est calculée par catégorie de niveau de satisfaction des besoins : bilans azotés nuls ou positifs, bilans énergétiques négatifs, ou positifs. Nous avons défini comme bilans azotés nuls tous ceux dont la valeur est comprise entre -1 et $+1 \mathrm{~g}$ d'azote par jour. 
Dans le cas des bilans azotés négatifs, l'azote digéré ne représente qu'une partie de l'azote utilisé, le complément étant fourni par diminution du pool azoté de l'organisme (azote mobilisé). Nous établissons donc l'équation de régression suivante :

$$
N P_{\text {lait }}=a N_{\text {digéré }}-b P^{0,75}+c N_{\text {biran }}
$$

\section{Résultats et discussion}

\section{A. Résultats (tabl. 3)}

Les chèvres consomment en moyenne $3,8 \mathrm{~kg}$ de $\mathrm{MS} / 100 \mathrm{~kg}$ de $\mathrm{PV}$. Le maximum: de consommation est obtenu pour des chèvres en $7^{\circ}$ semaine de lactation et le minimum pour une chèvre en $1^{\text {re }}$ semaine de lactation.

TABleau 3

Caractéristiques des chèvres, de leur consommation et de leur production.

Characteristics of the goats; their feed intake and milk production.

\begin{tabular}{|c|c|c|c|c|}
\hline & $\begin{array}{l}\text { Moyenne } \\
\text { Mean }\end{array}$ & $\begin{array}{l}\text { Ecart-type } \\
\text { Standard } \\
\text { deviation }\end{array}$ & Minimum & Maximum \\
\hline Poids - Weight $(\mathrm{kg}) \ldots \ldots \ldots \ldots$ & 47,5 & 5,0 & 35,6 & 63,4 \\
\hline MSVI - DMI $(\mathrm{kg} / \mathrm{j}-k g / d)$ & 1,8 & 0,5 & 0,7 & 2,8 \\
\hline $\mathrm{N}$ ingéré $-N$ intake $(\mathrm{g} / \mathrm{j}-g / d)$ & 41,1 & 12,0 & 13,6 & 67,3 \\
\hline $\mathrm{N}$ fécal - faecal $N(\mathrm{~g} / \mathrm{j}-g / d) \ldots$ & 13,8 & 4,3 & 4,3 & 25,9 \\
\hline $\mathrm{N} \operatorname{dig}-\operatorname{dig} N(\mathrm{~g} / \mathrm{j}-g / d) \ldots \ldots$ & 27,2 & 8,6 & 7,7 & 42,3 \\
\hline $\mathrm{N}$ urinaire - urinary $N(\mathrm{~g} / \mathrm{j}-g / d)$ & 8,9 & 3,5 & 2,8 & 19,3 \\
\hline Lait - milk $(\mathrm{kg} / \mathrm{j}-k g / d)$ & 2,7 & 0,9 & 0,3 & 5,0 \\
\hline NP lait - milk $P N(\mathrm{~g} / \mathrm{j}-g / d) \ldots$ & 12,8 & 3,8 & 2,1 & 22,5 \\
\hline $\begin{array}{l}\text { B:lan de l'énergie }(\mathrm{UFL} / \mathrm{j}) \ldots \ldots \\
\text { Energy balance }(U F L / d)\end{array}$ & $-0,15$ & 0,49 & $-1,47$ & 0,83 \\
\hline $\begin{array}{l}\text { Bilas de l'azote }(\mathrm{g} / \mathrm{j}) \ldots \ldots \ldots \ldots \\
\text { Nitrogen balance }(g / d)\end{array}$ & 4,51 & 5,54 & $-9,27$ & 17,3 \\
\hline $\begin{array}{l}\text { Stade de lactation (semaines) } \ldots . . . \\
\text { Lactation stage (weeks) }\end{array}$ & 7,8 & - & 1 & 40 \\
\hline $\begin{array}{l}\text { PDIN-PDIE ingérées }(\mathrm{g} / \mathrm{j}) \quad \ldots \ldots \\
P D I N-P D I E \text { intake }(g / d)\end{array}$ & $-23,7$ & 13,9 & -56 & 27 \\
\hline
\end{tabular}


Les productions laitières maximales sont obtenues en début de lactation $\left(5^{\circ} \mathrm{se}-\right.$ maine) alors que la production minimum est mesurée sur une chèvre en $40^{\circ}$ semaine de lactation.

\section{Tableau 4}

Coefficients de corrélation $(x 1000)$.

Correlation coefficients.

\begin{tabular}{|c|c|c|c|c|}
\hline $\begin{array}{ll}\begin{array}{l}\text { Bilans } \\
\text { Balances }\end{array} & \\
& \begin{array}{l}\text { Energie } \\
\text { Energy }\end{array} \\
& \begin{array}{l}\text { Azote } \ldots \ldots \ldots \ldots \\
\text { Nitrogen }\end{array} \\
\end{array}$ & $\begin{array}{l}<0 \\
<0\end{array}$ & $\begin{array}{l}<0 \\
>0\end{array}$ & $\begin{array}{l}>0 \\
>0\end{array}$ & $\begin{array}{c}\text { Ensemble } \\
\text { Total }\end{array}$ \\
\hline $\begin{array}{l}\mathrm{N} \text { dig. } \times \text { NP lait } \ldots \ldots \ldots \ldots \\
\text { dig. } N \times \text { milk } P N\end{array}$ & 709 & 683 & 735 & 481 \\
\hline $\begin{array}{l}\mathrm{N} \text { dig. } \times \mathrm{N} \text { bil. } \ldots \ldots \ldots \ldots \ldots \ldots \\
\text { dig. } N \times N \text { balance }\end{array}$ & 397 & 730 & 555 & 779 \\
\hline $\begin{array}{l}\text { NP lait } \times \mathrm{N} \text { bil. } \ldots \ldots \ldots \ldots \ldots \\
\text { milk } P N \times N \text { balance }\end{array}$ & -221 & 222 & 121 & -10 \\
\hline $\begin{array}{l}\mathrm{N} \text { bil. } \times \mathrm{P}^{0,75} \ldots \ldots \ldots \ldots \\
N \text { balance } \times W^{0.75}\end{array}$ & -406 & 153 & 320 & - \\
\hline $\begin{array}{l}\text { NP lait } \times P^{0.75} \ldots \ldots \ldots \ldots \ldots \\
\text { milk } P N \times W^{0.75}\end{array}$ & -52 & 222 & 202 & 275 \\
\hline 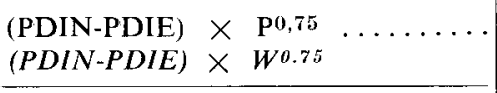 & -45 & -248 & -247 & -228 \\
\hline $\begin{array}{l}\text { NP lait } \times(\text { PDIN-PDIE }) \ldots \ldots \\
m i l k P N \times(P D I N-P D I E)\end{array}$ & -218 & -464 & -452 & -447 \\
\hline $\begin{array}{l}\text { MA ins. } \times \mathrm{N} \text { fécès } \ldots \ldots \ldots \\
\text { Ins. } P \times \text { faecal } N\end{array}$ & & & & 782 \\
\hline $\begin{array}{l}\text { MOD } \times \text { CBND } \ldots \ldots \ldots \ldots \ldots \\
D O M \times N D C F\end{array}$ & & & & 83 \\
\hline $\begin{array}{l}\text { Ma ins. } \times \text { CBND } \\
\text { Ins. } P \times N D C F\end{array}$ & & & & 355 \\
\hline $\begin{array}{l}\text { MOD } \times \mathrm{N} \text { fécès } \\
D O M \times \text { faecal } N\end{array}$ & & & & 716 \\
\hline
\end{tabular}


43 bilans azotés sont négatifs (tabl. 5). Les $2 / 3$ d'entre eux sont mesurés chez des chèvres en $1^{\text {re }}$ ou en $2^{\mathrm{r}}$ semaine de lactation. Le déficit azoté est expliqué par une faible consommation de matière sèche due le plus souvent à la proximité de la mise-bas (tabl. 5). Ces chèvres ont mobilisé en moyenne 3,8 g d'azote par animal et par jour pendant deux semaines, soit $55 \mathrm{~g}$ d'azote pour l'ensemble de la période. Cette mobilisation a atteint, chez une chèvre, $115 \mathrm{~g}$ d'azote en deux semaines. Toutes les chèvres en bilan azoté négatif sont aussi en bilan énergétique négatif. L'azote mobilisé est corrélé avec le poids métabolique des animaux (tabl. 4). Les chèvres les plus lourdes mobilisent davantage de réserves protéiques.

173 bilans azotés sont positifs ou nuls : 70 sont obtenus sur des animaux en bilan énergétique négatif et 103 sur des animaux en bilan énergétique positif ou nul. Les quantités d'azote digéré et d'azote protéique du lait sont toujours fortement corrélées $(r>0,68)$ alors que la quantité d'azote digérée n'est bien corrélée avec la quantité d'azote du bilan que dans le cas des animaux en bilan azoté positif $(r>0,5)($ tabl. 4).

\section{B. Calcul des besoins d'entretien par la méthode factorielle}

\section{Azote urinaire endogène}

Pour les chèvres en bilan azoté négatif ou nul ( $-2,8 \mathrm{~g}$ en moyenne) recevant une ration déficitaire en azote soluble (PDIN - PDIE $=-13 \mathrm{~g}$ en moyenne) l'équation obtenue est la suivante :

$$
\mathrm{N}_{\text {urinaire }}=0,315 \mathrm{~N}_{\text {digéré }}+0,126 \mathrm{PV}-4,21 \quad \begin{aligned}
& \mathrm{n}=54 \\
& i=0,67 \text { or }=2,24
\end{aligned}
$$

Dans cette équation, le terme constant n'est pas significativement différent de zéro. Nous établissons une équation n'incluant pas de terme constant :

$$
\begin{array}{ccc}
\mathrm{N}_{\text {urinaire }}=0,297 & \mathrm{~N}_{\text {digéré }}+0,0434 \mathrm{PV} & \mathrm{n}=54 \\
\sigma=0,05 & \sigma=0,02 & \mathrm{r}=0,96
\end{array}
$$

Nous admettons que, en cas d'apport d'azote digestible nul, EUN est égal à $0,0434 \mathrm{~g} / \mathrm{kg}$ PV. Cette valeur correspond à $0,111 \mathrm{~g} / \mathrm{kg} \mathbf{P}^{0,75}$. Elle est identique à celles obtenues sur boucs castrés par RAJPOOT, SENGAR \& SingH (1980) $(0,11$ à $\left.0,12 \mathrm{~g} / \mathrm{kg} \mathrm{P}^{0,75}\right)$ et par Blanchart \& BRUN-Bellut (1982) $\left(0,113 \mathrm{~g} / \mathrm{kg} \mathrm{P}^{0 / 75}\right)$, mais un peu plus faible que celles obtenues par DevendRA $(1980)$ sur chèvres $(0,133 \mathrm{~g} / \mathrm{kg}$. $\left.\mathrm{P}^{0,75}\right)$.

\section{Azote fécal métabolique}

L'équation obtenue à partir du modèle proposé est la suivante :

$$
\mathrm{N}_{\text {Féc }}=0,0171 \mathrm{MA}_{\text {ins. }}+0,00606 \mathrm{MOD}+0,0169 \mathrm{CBND}
$$

et la production d'azote fécal métabolique est :

$$
\mathrm{MFN}=0,0169 \mathrm{CBND}
$$

Dans notre étude la valeur prise par MFN est 2,57 g N/animal/jour, soit

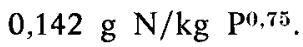




\section{TABleau 5}

Utilisation de l'azote selon l'état nutritionnel des chèvres.

Nitrogen utilization according to the nutritional status of the goats.

\begin{tabular}{|c|c|c|c|c|c|}
\hline \multirow{2}{*}{$\begin{array}{l}\text { Bilan } \\
\text { Balance }\end{array}$} & $\begin{array}{l}\text { Energie } \\
\text { Energy }\end{array}$ & $<0$ & $<0$ & $<0$ & $>0$ \\
\hline & $\begin{array}{l}\text { Azote } \\
\text { Nitrogen }\end{array}$ & $<0$ & $=0$ & $>0$ & $>0$ \\
\hline \multicolumn{2}{|l|}{$\begin{array}{l}\text { Nombre } \\
\text { Number }\end{array}$} & 43 & 20 & 70 & 103 \\
\hline \multicolumn{2}{|c|}{$\begin{array}{l}\text { Stade (semaines) } \\
\text { Stage (weeks) }\end{array}$} & 2 & 2,5 & 3,5 & 12,5 \\
\hline \multicolumn{2}{|l|}{$\begin{array}{l}\mathrm{N} \text { dig. }(\mathrm{g} / \mathrm{j}) \\
\operatorname{dig} . N(g / d)\end{array}$} & 17,3 & 21,7 & 27,0 & 31,6 \\
\hline \multicolumn{2}{|c|}{$\begin{array}{l}\text { Lait standard }(\mathrm{kg}) \\
\text { Standard milk }(\mathrm{kg})\end{array}$} & 2,80 & 3,02 & 3,10 & 2,40 \\
\hline \multicolumn{2}{|c|}{$\begin{array}{l}\text { Bilan de l'azote }(\mathrm{g} / \mathrm{j}) \\
\text { Nitrogen balance }(g / d)\end{array}$} & $-3,8$ & 0 & 4,1 & 8,2 \\
\hline \multicolumn{2}{|c|}{$\begin{array}{l}\text { Bilan de l'énergie (UFL/j) } \\
\text { Energy balance }(U F L / d)\end{array}$} & $-0,80$ & $-0,53$ & $-0,40$ & 0,26 \\
\hline \multicolumn{2}{|c|}{$\begin{array}{l}* \text { Excès } \mathrm{N}\left(\mathrm{g} / \mathrm{j} / \mathrm{kg} \mathrm{P}^{0,75}\right) \\
* N \text { excess }\left(g / d / \mathrm{kg} W^{0.75}\right)\end{array}$} & 0,20 & 0,36 & 0,64 & 1,05 \\
\hline \multicolumn{2}{|c|}{$\begin{array}{l}\mathrm{N} \text { urine }(\mathrm{g} / \mathrm{j}) \\
\text { Urine } N(g / d)\end{array}$} & 7,5 & 7,0 & 7,2 & 10,6 \\
\hline
\end{tabular}

$*$ Excès $\mathrm{N}=\frac{\mathrm{N} \text { Dig. }-\mathrm{N} \text { Lait }}{\mathrm{P} 0,75} ; N$ Excess $=\frac{\text { Dig. } N-\text { Milk } N}{W 0.7 \bar{s}}$

Nos résultats sont inférieurs à ceux obtenus par DEvENDra (1980) sur chèvres $\left(0,221 \mathrm{~g} / 100 \mathrm{~g}\right.$ de MS ingérée, valeur qui correspondrait ici à $\left.0,22 \mathrm{~g} \mathrm{~N} / \mathrm{kg} \mathrm{P}^{0,75}\right)$ ainsi qu'à ceux de Rajpoot, Sengar \& Singh (1980) $(0,43 \mathrm{~g} / 100$ de MS ingérée, valeur qui correspondrait ici à $0,43 \mathrm{~g} \mathrm{~N} / \mathrm{kg} \mathrm{P}^{0.75}$ ) et de BLANCHART \& BRUN-Bellut (1982) $\left(0,21\right.$ à $\left.0,23 \mathrm{~g} \mathrm{~N} / \mathrm{kg} \mathrm{P}^{0 / 7 \pi}\right)$ sur boucs castrés.

\section{Besoins azotés d'entretien}

Les pertes totales d'azote endogène sont donc :

$$
\mathrm{EUN}+\mathrm{MFN}=0,0169 \mathrm{CBND}+0,0434 \mathrm{PV}
$$

Calculées à partir des valeurs moyennes mesurées, ces pertes qui correspondent au besoin d'entretien sont de $0,25 \mathrm{~g} \mathrm{~N} / \mathrm{kg} \mathrm{P}^{0,75}$. 


\section{Calcul des besoins azotés par la méthode globale}

1. Chèvres en bilan azoté nul et en bilan énergétique négatif

20 bilans ont été mesurés sur des animaux dans cette situation (tabl. 5). Il s'agit de chèvres le plus souvent en $2^{\circ}, 3^{\circ}$ et $4^{\circ}$ semaine de lactation.

$$
\begin{array}{cccc}
\mathrm{N}_{\text {digéré }}=1,38 \mathrm{NP}_{\text {lait }}+0,32 & \mathrm{P}^{0 / 75}+0,11 \Delta \text { PDI } & \mathrm{n}=20 \\
\sigma=0,25 & \sigma=0,16 & \sigma=0,05 & \mathrm{r}=0,99
\end{array}
$$

Les termes $\mathrm{N}$ bilan et TUL n'entrent pas significativement dans l'équation.

Les variables $N P_{1 a i t}$, $P^{0} \tau 5$ et $\Delta$ PDI n'étant que peu liées (tabl. 4) leurs coefficients dans cette équation peuvent être utilisés séparément. Le rendement de transformation de l'azote digéré en azote protéique du lait est de $1 / 1,38$ soit 0,72 et les besoins d'entretien sont de $0,32 \mathrm{~g} \mathrm{~N}_{\mathrm{dig}} / \mathrm{kg} \mathrm{P}^{0,75}$.

\section{Chèvres en bilan azoté positif et en bilan énergétique négatif}

70 bilans ont été mesurés sur des animaux dans cette situation (tabl. 5). Il s'agit de chèvres en $3^{\circ}$ ou en $4^{\circ}$ semaine de lactation.

$$
\begin{gathered}
\mathrm{N}_{\text {digéré }}=1,23 \mathrm{NP}_{\text {lait }}+0,32 \mathrm{P}^{0 / \tau}+0,07 \Delta \text { PDI }+1,24 \quad \mathrm{~N}_{\text {lila }} \quad \mathrm{r}=0,998 \\
\sigma=0,11 \quad \sigma=0,08 \quad \sigma=0,03 \quad \sigma=0,10
\end{gathered}
$$

La variable TUL n'entre pas significativement dans l'équation. Le rendement de transformation de l'azote digéré en azote protéique du lait est ici de 1/1,23 soit 0,81 et les besoins d'entretien sont de $0,32 \mathrm{~g} \mathrm{~N}_{\text {digéré }} / \mathrm{kg}$ P0ris

\section{Chèvres en bilan azoté positif et bilan énergétique positif ou nul}

103 bilans azotés ont été mesurés sur des animaux dans cette situation (tabl. 5). En moyenne, les chèvres se trouvent en $13^{\circ}$ ou $14^{\circ}$ semaine de lactation ; cependant, l'amplitude est importante puisque certaines sont en $2^{\circ}$ ou en $3^{\prime \prime}$ semaine et d'autres sont en $40^{\circ}$ semaine de lactation.

Pour l'ensemble de ces mesures, l'excès d'azote digéré par rapport à l'exportation d'azote dans le lait est élevé (tabl. 5) : la consommation d'azote est maximale, alors que la production laitière est plus faible que dans les autres cas. Le taux d'urée du lait permettant une estimation de cet excès d'azote entre donc dans l'équation :

$$
\begin{aligned}
\mathrm{N}_{\text {digéré }}=1,28 \quad \mathrm{NP}_{\text {lait. }}+0,32 \quad \mathrm{P}^{\prime} \mathrm{T} 5+0,83 \quad \mathrm{~N}_{\text {bilan }}+0,09 \Delta \quad \mathrm{PDI}+18,59 \text { TUL } \\
\sigma=0,07 \quad \sigma=0,11 \quad \sigma=0,08 \quad \sigma=0,03 \quad \sigma=4,27 \quad \mathrm{r}=0,997
\end{aligned}
$$

Les besoins d'entretien sont de $0,32 \mathrm{~g} \mathrm{~N} / \mathrm{kg} \mathrm{P}^{0,75}$ et le rendement de la transformation de l'azote digéré en azote protéique du lait est de 1/1,28 soit 0,78.

La faible valeur du coefficient de $\mathrm{N}$ bilan $(0,83)$ peut être en partie expliquée par son mode de détermination. Les imprécisions de mesure de l'azote excrété au niveau des fèces, des urines et du lait sont faibles au niveau de chacun des constituants mais s'additionnent pour surestimer $\mathrm{N}$ bilan. L'erreur peut être importante 
dans la mesure où $\mathrm{N}$ bilan est proche de zéro. La variable $\mathrm{N}$ bilan n'étant pas corrélée aux variables $\mathrm{P}^{0 / 75}$ et NP lait, nous pouvons admettre que cette surestimation est compensée dans l'équation par une diminution du coefficient de $\mathrm{N}$ bilan : ceci ne nous permet pas d'estimer le rendement de la fixation de l'azote.

\section{Ensemble des chèvres en bilan azoté positif ou nul}

Pour l'ensemble des mesures effectuées sur des chèvres en bilan azoté positif ou nul, les besoins d'entretien s'élèvent à $0,32 \mathrm{~g} / \mathrm{kg} \mathbf{P}^{075}$. Le rendement de transformation de l'azote digéré en azote protéique du lait varie entre 0,72 (bilan azoté nul et bilan énergétique négatif) et 0,81 (bilan azoté positif et bilan énergétique négatif). La valeur la plus élevée est obtenue dans le cas des chèvres en bilan azoté légèrement positif et en bilan énergétique légèrement négatif.

Dans le cas des bilans azotés nuls et des bilans énergétiques négatifs, il est possible que des acides aminés soient dégradés pour produire de l'énergie ou que la synthèse des protéines du lait soit affectée par la carence en énergie de l'organisme. Ce phénomène tend à disparaître dans le cas des bilans azotés légèrement positifs et des bilans énergétiques négatifs.

Il est vraisemblable que le rendement optimum se situe dans le cas des animaux en bilans azoté et énergétique nuls.

\section{Animaux en bilan azoté négatif}

Nous avons regroupé 43 mesures de bilans azotés négatifs (tabl. 5). La variable $\mathrm{P}^{0 / 75}$ n'est pas significativement liée à la variable $\mathrm{NP}_{\text {lait }}(\mathrm{r}=0,003)$; elle n'entre pas significativement dans l'équation obtenue d'après le modèle proposé.

L'équation de régression retenue est donc :

$$
\begin{aligned}
& \mathrm{NP}_{\text {lait }}=0,56 \mathrm{~N}_{\text {digéré }}+0,71 \mathrm{~N}_{\text {mobilisé }} \\
& \sigma=0,02 \quad \sigma=0,06 \quad \mathbf{r}=0,995
\end{aligned}
$$

Le rendement de l'azote digéré en azote protéique du lait est plus faible que ceux obtenus dans les autres cas. Cet écart peut être lié à la différence entre les modèles des équations utilisées pour calculer le rendement. Il peut aussi être provoqué par les conditions nutritionnelles dans lesquelles se trouvent les animaux ; en particulier, lorsque les chèvres sont en bilan azoté négatif, elles présentent le bilan énergétique le plus déficitaire (tabl. 5). La faiblesse du rendement de transformation de l'azote digéré en azote protéique du lait pourrait être due à l'utilisation d'une partie des protéines digérées pour la fourniture d'énergie à l'animal.

Le rendement de la transformation de l'azote mobilisé en azote protéique du lait est plus élevé $(0,71)$ ce qui n'est peut-être dû qu'à une sous-estimation de l'azote mobilisé, liée à la méthode de détermination de $\mathrm{N}$ bilan. 


\section{Conclusion}

Les besoins d'entretien des chèvres en lactation déterminés par la méthode factorielle sont un peu inférieurs à ceux déterminés par la méthode globale $(0,25$ et $0,31 \mathrm{~g} / \mathrm{kg} \mathrm{P}^{0 / 75}$ respectivement). Les valeurs obtenues correspondent à des besoins de 1,56 à $2 \mathrm{~g}$ de MAD/kg $\mathrm{P}^{0,75}$ soit 60 à $75 \mathrm{~g}$ de MAD/100 $\mathrm{kg}$ PV. Cette différence entre les deux méthodes a déjà été observée de manière encore plus nette par Majumdar (1960) $(+60$ p. 100) et par Sengar en $1980(+100$ p. 100) (tabl. 1). Dans nos conditions de mesures, la différence pourrait être expliquée, au moins partiellement, par une sous-estimation de EUN par la méthode de régression utilisée. En effet, nous avons admis que pour des animaux en bilan azoté négatif ou nul l'équation de régression expliquant l'azote urinaire par l'azote digéré était linéaire. Il est possible que d'autres modèles fournissent des valeurs de EUN supérieures.

D'autre part, les pertes d'azote urinaire sont difficiles à éviter dans les mesures de bilan azoté. Ces pertes se répercutent dans l'équation obtenue soit sur le coefficient de la variable Ndig soit sur celui du poids vif, entraînant ainsi une sousestimation de EUN et des besoins d'entretien.

En moyenne sur l'ensemble de la lactation, les rendements de transformation de l'azote digéré en azote protéique du lait que nous avons obtenus $(0,56$ à 0,81$)$ sont peu différents de ceux mesurés sur vaches laitières par TAMminga \& OLdham en $1980(0,60$ à 0,80$)$ et sur chèvres (tabl. 1). Les régimes que nous avons utilisés ont sans doute joué un rôle favorable dans l'obtention de ces rendements élevés. En particulier, le gaspillage d'azote au niveau du rumen a probablement été limité par la relative «pauvreté » en azote soluble des rations, caractérisće par la valeur PDIN-PDIE (tabl. 3). Les niveaux d'azote excrété dans l'urine (en moyenne de $8,9 \mathrm{~g} /$ jour) inférieurs aux valeurs mesurées par CisuK (1980) et Daccond (1981) confortent cette hypothèse.

Il serait intéressant de connaître exactement les possibilités qu'ont les chèvres d'économiser et de recycler de l'azote sans altération de la digestibilité de la ration ni des performances de l'animal.

\section{Summary \\ Determination of protein requirements in lactating dairy goats}

Protein requirements of lactating goats were determined using 216 individual nitrogen balances measured in 67 goats between the 1 st and the 40 th week after kidding (table 3 ).

Two methods were employed :

1) Factorial method: The protein requirement for maintenance was considered as the sum of endogenous urinary nitrogen (EUN) and metabolic faecal nitrogen (MFN) : EUN $=0.0434 \mathrm{~g} \mathrm{~N} / \mathrm{kg} \mathrm{LW} ; \mathrm{MFN}=0.0169 \mathrm{~g} \mathrm{~N} / \mathrm{g}$ of Non Digestible Crude Fibre intake.

In our trials minimum nitrogen losses were EUN $+\mathrm{MFN}=0.25 \mathrm{~g} \mathrm{~N} / \mathrm{kg} \mathrm{W} \mathrm{W}^{0.75}$.

2) Overal method : Digestible nitrogen represents : nitrogen for maintenance (proportional to metabolic weight), for milk protein production (proportional to milk protein 
nitrogen) for increasing nitrogen pool (proportional to nitrogen balance); a part is lost in rumen metabolism (proportional to the difference between PDIN and PDIE), and in urine when the level of dietary nitrogen is higher than milk protein production (proportional to milk urea content).

Protein requirements for maintenance and milk production were calculated by repression. Nitrogen requirement for maintenance equals $0.32 \mathrm{~g} \mathrm{~N} / \mathrm{kg} \mathrm{W0.75}$. The efficiency of digestible nitrogen to produce milk protein nitrogen was between 0.72 and 0.81 when nitrogen and energy balances varied (tables 4 and 5). The highest efficiencies were obtained with a positive nitrogen and a negative energy balance.

These high values could be explained by a nitrogen saving (urinary nitrogen was low: $8.9 \mathrm{~g} \mathrm{~N}$ per day).

In nitrogen deficient animals, the efficiency of digestible nitrogen to produce milk protein was equal to 0.56 . It was lower than the efficiency of body nitrogen to produce milk protein nitrogen $(0.71)$.

Key words : nitrogen, goat, maintenance, milk.

Reçu en avril 1983.

Accepté en décembre 1983.

\section{Références bibliographiques}

Agricultural Research Council 1965. The nutrient requirements of farm live-stock, 2. Ruminants, 153-192, ed. A.R.C., London.

Akinsoyinu A.O., 1981. Protein metabolism and requirements, 127-136. In : Nutrition et systèmes d'alimentation, 1. Symposium international, Tours, INRA-ITOVIC ed., Paris.

Blanchart G., Brun-Bellut J., 1982. Determination of protein maintenance requirements in goats from nitrogen losses in urine and faeces. Communication, projet $B$ « Nutrition des caprins », sous-réseau FAO, Reading 9-10 novembre 1982.

Blanchart G., Brun-Bellut J., Vignon B., 1980. Comparaison des caprins aux ovins quant à l'ingestion, la digestibilité et la valeur alimentaire de diverses rations. Reprod. Nutr. Dev., 20, 1731-1737.

Blanchart G., Brun-Bellut J., Vignon B., 1983. L'azote fécal chez la chèvre laitière : relations avec la digestion des aliments et détermination de l'azote fécal métabolique. IV Symp. Int. Métabolisme et nutrition azotés, Clermont-Ferrand (France), 2, 165168, Ed. I.N.R.A. Publ. (les Colloques de l'I.N.R.A., $\mathrm{n}^{\circ} 16$ ).

Brun-Bellut J., Laurent F., Vignon B., 1983. Taux d'urée du lait et utilisation de l'azote par la chèvre laitière. IV Symp. Int. Métabolisme et nutrition azotés, Clermont-Ferrand (France), 2, 179-182. Ed. I.N.R.A. Publ. (les Colloques de l'I.N.R.A., $\left.\mathrm{n}^{\circ} 16\right)$.

CisuK P., 1980. Nitrogen balance and digestibility in lactating goats on rations with varied nitrogen and energy sources. Rapport $n^{\circ} 53, p$. 46. Swedish Univ. Agricultural Sciences, Dep. Animal Nutrition. Uppsala, Sweden.

DACCORD R., 1981. Influence du mode de conservation de l'herbe sur le métabolisme azoté de la chèvre, 168-179. In : Nutrition et systèmes d'alimentation, 1. Symposium International. Tours, INRA-ITOVIC Ed., Paris.

DEVEndRA C., 1980. The protein requirements for maintenance of indigenous Kambing Katpang goats in Malaysia. Nutr. Abs. Rev. 52, 2347 (Abstr.).

Giger S., Sauvant D., 1983. Etude des facteurs de variation de l'excrétion azotée fécale chez la chèvre. IV $V^{e}$ Symp. Int. métabolisme et nutrition azotés, Clermont-Ferrand (France), 2, 169-173. Ed. I.N.R.A. Publ. (les Colloques de l'I.N.R.A., ${ }^{\circ}{ }^{16}$ ).

Itoch M., HaRvy T., TaNo R., IWASAKI K., 1978. Maintenance requirements of energy and protein for castrated Japanese native goats. Nutr. Abstr. Rev. 49, 142 (Abstr.). 
KAUFMANN W., 1976. Influence of the composition of the ration and the feeding frequency on $\mathrm{pH}$ regulation of rumen and on feed intake in ruminants. Livest. Prod. Sci., 3, 103-114.

Khouri S., 1974. Contribution à l'étude de l'évaluation de l'efficacité nutritive des protéines tannées chez le ruminant (cas particulier de la chèvre laitière). Thèse 3" cycle, Univ. des Sciences et Techniques du Languedoc, p. 109.

Majumdar B.N., 1960. Studies on goat nutrition. I. Minimum protein requirement of goats for maintenance. Endogenous urinary nitrogen and metabolic faecal nitrogen excretion studies. II. Digestible protein requirement for maintenance from balance studies. J. Agric. Sci., 34, 329-340.

Morand-Fehr P., Sauvant D., 1978. Caprins. In : R. Jarrige, Alimentation des ruminants, 449-467. I.N.R.A., 78000 Versailles.

Mugdal V.D., SiNGH N., 1981. Nitrogen excretion and protein requirements, 142-151. In : Nutrition et systèmes d'alimentation, 1. Symposium International, Tours, INRAITOVIC ed., Paris.

Rajpoot R.L., Sengar O.P.S., Singh S.N., 1980. Goats : protein requirement for maintenance. Int. Goat Sheep Res., 1, 182-189.

RAJPOOT R.L., 1981. Energy and protein in goat nutrition, 101-124. In : Nutrition et systèmes d'alimentation, 1. Symposium international, Tours, INRA-ITOVIC ed., Paris.

Reynolds L., 1981. Nitrogen metabolism in indigenous malawi goats, J. Agric. Sci., 96, 347-351.

SENGar O.P.S., 1980. Indian research on protein and energy requirements of goats. J. Dairy Sci., 63, 1655-1670.

Tamminga S., Oldham J.D., 1980. Amino acid utilization by dairy cows. II. Concept of amino acid requirements. Livest. Prod. Sci., 7, 453-463.

Verite R., Journet M., 1978. Vaches laitières. In : R. Jarrige, Alimentation des ruminants, 345-376, I.N.R.A., 78000 Versailles. 